\title{
Nutrition Screening and Assessment: A Critical Element of Care in Children with Developmental Disabilities and Special Health Care Needs
}

\section{Tina Crook ${ }^{1}$ and Reza Hakkak ${ }^{1-3}$}

${ }^{1}$ Department of Dietetics and Nutrition, University of Arkansas for Medical Sciences, 4301 W. Markham St., Little Rock, AR 72205, USA

${ }^{2}$ Department of Pediatrics, University of Arkansas for Medical Sciences, 4301 W. Markham St., Little Rock, AR 72205, USA

${ }^{3}$ Arkansas Children's Hospital Research Institute, 13 Children's Way, Little Rock, AR 72202, USA

The Centers for Disease Control defines developmental disabilities (DDs) as "a diverse group of severe chronic conditions that are due to mental and/or physical impairments" [1]. Individuals with DDs may face challenges learning, communicating, and/or physically caring for themselves. Some examples of DDs include, but are not limited to, cerebral palsy, autism spectrum disorders, intellectual disabilities and attention deficit hyperactivity disorder. Children with special health care needs (SCHNs) is a broader term that was defined by McPherson et al. [2] and captures individuals "who have or are at increased risk for a chronic physical, developmental, behavioral, or emotional condition and who also require health and related services of a type or amount beyond that required by children generally." Asthma and diabetes are examples of conditions included in this more inclusive definition.

The number of children with DDs has gradually increased over the past decade. The National Health Interview Surveys reported an increase from $12.84 \%$ in 1997 to $17.1 \%$ in 2008 [3]. Additionally, data from the 2009-2010 National Survey of Children with Special Heath Care Needs reported that $23 \%$ of households with children have at least one child with a special health care need, an increase of about $1.2 \%$ since the last reporting period [4].

Children with DDs and SHCNs are often at nutritional risk for a variety of reasons. They have unique nutrition challenges such as gastrointestinal disorders; poor growth; difficulties sucking, swallowing and chewing; inability to self-feed; textural issues with foods; and food avoidance. They are also at risk for developing secondary conditions such as obesity and endocrine disorders [5]. Schieve et al. [6] reported several concurrent medical conditions in children with DDs that are nutrition related including food allergies, diarrhea, colitis and stomach and intestinal illnesses. These problems were especially prevalent in children with autism.

Early nutrition interventions result in cost-savings and better outcomes for these children. For example, a malnourished child will be tired, lethargic and have trouble focusing. This will have a negative effect on the child's ability to perform in therapy sessions and the classroom. On the other hand, if the nutrition needs of the child are met, these barriers go away and the goals set for the child can be achieved, thus saving time, money and preventing frustration for the child and family.

Unfortunately, the importance of nutrition interventions in this population are often overlooked or given a low priority. Caregivers often feel overwhelmed because of the multiple medical and/or behavioral needs of their children. Thus, nutrition problems tend to go unnoticed or caregivers simply feel as if they cannot address the nutrition problems until the other issues are under control. Additionally, caregivers may not recognize how the child's current nutrition status influences their long-term outcomes.

The staff members in outpatient clinics and schools that routinely work with these children may also be unaware of the vital role that nutrition plays in the children's progress. It is imperative that staff members receive training in this area so they can reinforce this with the families. This illustrates the importance of an inter-professional team approach for managing the multiple aspects of care for children with DDs and SHCNs. A registered dietitian (RD) is an essential part of that team; however, it is unlikely that the outpatient clinics and schools these children attend have a RD on staff. Therefore, it is crucial that RDs are consulted to work with staff members in these facilities to establish ongoing nutrition screening processes, train staff and conduct thorough assessments on the children identified at nutritional risk.

A RD should work with facilities to establish appropriate nutrition screening processes to meet the unique needs of the population they serve. Nutrition screening must go beyond collecting anthropometric data on the children. We previously reported, children may plot within the normal ranges on growth charts yet still have micronutrient deficiencies [7]. Therefore, dietary intake must be considered by including standard questions that screen for dietary adequacy. The nutrition screen should also include medication usage since long-term use of some medications may result in medication-nutrient interactions [5]. Other factors that may be part of the nutrition screen include, but are not limited to, the family's availability to food, concurrent medical problems, food allergies/intolerances, supplement use and chewing or swallowing problems.

The RD should train staff members, including other healthcare professionals, on the importance of adequate nutrition in this population. Some of the basic concepts that should be addressed in training sessions include the importance of collecting accurate, routine anthropometric data; interpreting anthropometric data; recognizing physical signs of malnutrition; the negative impact of under-nutrition as well as over-nutrition; and using non-food rewards with the children.

In conclusion, nutrition must be recognized as a top priority by all healthcare team members and staff that work with children with DDs and SHCNs. Many of these children are at nutritional risk and require early nutrition interventions in order to achieve the best outcomes possible. $\mathrm{RDs}$ are an integral part of the inter-professional team caring for these children and should be included in their overall care.

\section{References}

1. Trends in the Prevalence of Developmental Disabilities in U. S. Children 19972008. CDC.

2. McPherson M, Arango P, Fox H, Lauver C, McManus $M$ et al. (1998) A new definition of children with special health care needs. Pediatrics 102: 137-140.

*Corresponding author: Dr. Reza Hakkak, Department of Dietetics and Nutrition, 4301 W. Markham St., Mail Slot 627, Little Rock, AR 72205, USA, Tel: 501-6866166; Fax: 501-686-5716; Email: HakkakReza@uams.edu

Received October 01, 2013; Accepted October 03, 2013; Published October 07 2013

Citation: Tina Crook, Hakkak R (2013) Nutrition Screening and Assessment A Critical Element of Care in Children with Developmental Disabilities and Special Health Care Needs. J Nutr Disorders Ther 3: e111. doi:10.4172/2161 $0509.1000 \mathrm{e} 111$

Copyright: ( $) 2013$ Tina Crook, et al. This is an open-access article distributed under the terms of the Creative Commons Attribution License, which permits unrestricted use, distribution, and reproduction in any medium, provided the original author and source are credited. 
Citation: Tina Crook, Hakkak R (2013) Nutrition Screening and Assessment: A Critical Element of Care in Children with Developmental Disabilities and Special Health Care Needs. J Nutr Disorders Ther 3: e111. doi:10.4172/2161-0509.1000e111

Page 2 of 2

3. Boyle C, Boulet S, Schieve L, Cohen R, Blumberg S et al. (2011) Trends in the prevalence of developmental disabilities in US Children, 1997-2008. Pediatrics 127: 1034-1042.

4. (2013) A project of the Child and Adolescent Health Measurement Initiative. CAHMI.

5. Van Riper CL, Wallace LS; American Dietetic Association (2010) Position of the American Dietetic Association: Providing nutrition services for people with developmental disabilities and special health care needs. J Am Diet Assoc110: 296-307.
6. Schieve LA, Gonzales V, Boulet SL, Visser SN, Rice CE, et al. (2012) Concurrent medical conditions and health care use and needs among children with learning and behavioral developmental disabilities, National Health Interview Survey, 2006-2010. Res Dev Disabil 33: 467-476.

7. Moore E, Crook T, James J, Gonzales D, Hakkak R (2012) Nutrient intake among children with autism. J Nutr Disorders Ther 2: 1-3. 